\title{
Implantable cardioverter-defibrillators in patients with long QT syndrome: a multicentre study
}

\author{
Agnieszka Zienciuk-Krajka ${ }^{1}$, Maciej Sterliński ${ }^{2}$, Artur Filipecki ${ }^{3}$, Radosław Owczuk ${ }^{4}$, Jacek Bednarek ${ }^{5}$, \\ Maciej Kempa ${ }^{1}$, Sławomir Sielski ${ }^{6}$, Marcin Dziduszko ${ }^{7}$, Przemysław Mitkowski ${ }^{8}$, Jarosław Kaźmierczak ${ }^{9}$, \\ Jacek Kuśnierz ${ }^{10}$, Dariusz Michałkiewicz ${ }^{11}$, Sebastian M. Stec ${ }^{12,}{ }^{13}$, Marcin Gułaj ${ }^{14}$, Ryszard Majcherek ${ }^{15}$, \\ Andrzej Lubiński ${ }^{16}$, Grzegorz Raczak ${ }^{1}$
}

'Department of Cardiology and Electrotherapy, Medical University of Gdansk, Gdansk, Poland

${ }^{2} 2^{\text {nd }}$ Department of Coronary Artery Disease, Institute of Cardiology, Warsaw, Poland

${ }^{3} 1^{\text {st }}$ Department of Cardiology, Silesian Medical University, Katowice, Poland

${ }^{4}$ Department of Anaesthesiology and Intensive Therapy, Medical University of Gdansk, Gdansk, Poland

${ }^{5}$ Department of Electrocardiology, Jagiellonian University, Krakow, Poland

${ }^{6}$ Department of Cardiology, Nicolaus Copernicus University, Bydgoszcz, Poland

'Department of Cardiology, Medical University, Lublin, Poland

${ }^{8} 1^{\text {st }}$ Department of Cardiology, Medical University, Poznan, Poland

${ }^{9}$ Department of Cardiology, Pomeranian Medical University, Szczecin, Poland

${ }^{10}$ Department of Cardiology, MEDICOVER Hospital, Warsaw, Poland

${ }^{11}$ Department of Cardiology, Institute of Cardiology, Bielanski Hospital, Warsaw, Poland

${ }^{12}$ Department of Cardiology, Postgraduate Medical School, Warsaw, Poland

${ }^{13}$ Department of Medicine, University of Rzeszow, Rzeszow, Poland

${ }^{14}$ Department of Cardiology, Hospital of the Ministry of Internal Affairs, Bialystok, Poland

${ }^{15}$ Department of Cardiology, Specialist Hospital, Tarnow, Poland

${ }^{16}$ Department of Invasive Cardiology and Cardiodiabetology, Medical University of Lodz, Lodz, Poland

\section{Abstract}

Background: Implantable cardioverter-defibrillator (ICD) therapy has been proven effective in the prevention of sudden cardiac death, but data on outcomes of ICD therapy in the young and otherwise healthy patients with long QT syndrome (LQTS) are limited. Aim: We sought to collect data on appropriate and inappropriate ICD discharges, risk factors, and ICD-related complications. Methods: All LQTS patients implanted with an ICD in 14 centres were investigated. Demographic, clinical, and ICD therapy data were collected.

Results: The study included 67 patients (88\% female). Median age at ICD implantation was 31 years (12-77 years). ICD indication was based on resuscitated cardiac arrest in 46 patients, syncope in 18 patients, and malignant family history in three patients. During a median follow-up of 48 months, 39 (58\%) patients received one or more ICD therapies. Time to first appropriate discharge was up to 55 months. Inappropriate therapies were triggered by fast sinus rhythm, atrial fibrillation, and T-wave oversensing. No predictors of inappropriate shocks were identified. Risk factors for appropriate ICD therapy were: (1) recurrent syncope despite $\beta$-blocker treatment before ICD implantation, (2) pacemaker therapy before ICD implantation, (3) single-chamber ICD, and (4) noncompliance to $\beta$-blockers. In 38 (57\%) patients, at least one complication occurred.

Conclusions: ICD therapy is effective in nearly half the patient population; however, the rates of early and late complications are high. Although the number of unnecessary ICD shocks and reimplantation procedures may be lowered by modern programming and increased longevity of newer ICD generators, other adverse events are less likely to be reduced.

Key words: device recalls, ICD outcome, implantable cardioverter-defibrillator, lead dysfunction, long QT syndrome, risk factors

Kardiol Pol 2018; 76, 12: 1687-1696

Agnieszka Zienciuk-Krajka, MD, PhD, Department of Cardiology and Electrotherapy, Medical University of Gdansk, ul. Dębinki 7, 80-952 Gdańsk, Poland, e-mail: agzien@gumed.edu.pl

Received: 12.03.2018 Accepted: 16.08.2018

Available as AoP: 17.08 .2018

Kardiologia Polska Copyright (C) Polish Cardiac Society 2018 


\section{INTRODUCTION}

Inherited non-syndromic long QT syndrome (LQTS) is characterised by QT interval prolongation on the surface electrocardiogram and a risk for life-threatening ventricular arrhythmias in the absence of structural heart disease. In the context of LQTS, general agreement exists that patients presenting with aborted sudden death have a higher risk of recurrent arrhythmic events and should receive an implantable cardioverter-defibrillator (ICD), whereas more controversy surrounds ICD implantation in the primary prevention of sudden cardiac death (SCD) [1, 2].

No randomised clinical trial has ever been conducted on ICDs in LQTS patients, thus registry data on outcomes of ICD therapy in the young and otherwise healthy population of LQTS patients are still of importance. We conducted a multicentre study to collect information on appropriate and inappropriate ICD therapies, risk factors, and complication rates in the long-term follow-up of primary and secondary preventive ICD therapy in LQTS patients.

\section{METHODS \\ Patient population}

Patients were included in the study if they had a documentation of corrected QT interval (QTc) prolongation $>450 \mathrm{~ms}$ (men) and $>460 \mathrm{~ms}$ (women) in the absence of QT-prolonging drugs and if they met at least one of the following criteria [3]: (i) history of aborted SCD; (ii) history of recurrent syncope with or without concomitantly recorded torsade de pointes (TdP); (iii) malignant family history of sudden death ( $\geq$ two sudden deaths in close relatives $<40$ years old).

A total of 74 patients referred as suspected LQTS cases were screened. Of these, seven patients were excluded because they were subsequently diagnosed with polymorphic catecholaminergic ventricular tachycardia $(\mathrm{VT} ; \mathrm{n}=3)$, myocarditis $(n=2)$, drug-induced QT prolongation $(n=1)$, and idiopathic ventricular fibrillation $(\mathrm{VF} ; \mathrm{n}=1)$. The remaining 67 patients were included in further analysis.

The following clinical data were collected in all 14 participating centres: circumstances of diagnosis and indications for ICD implantation, age at first symptoms and at diagnosis, sex, family history of LQTS and SCD ( $<40$ years of age), and the efficacy of previous treatment. Left cardiac sympathetic denervation was not performed in any patient.

\section{Electrocardiographic definitions}

Long QT syndrome was diagnosed when QTc interval prolongation > $440 \mathrm{~ms}$ in men and > $460 \mathrm{~ms}$ in women was recorded in the absence of QT-prolonging drugs. QT measurements were made in leads II and V5. Corrections were made using Bazett's formula.

\section{ICD implantation}

A single- or dual-chamber ICD was implanted since December 1995 according to the investigator's preference. The decision was based on the presence of concomitant pacing indication and/or on the presence of supraventricular arrhythmias. Implantation was performed with a non-thoracotomy transvenous lead system in all patients. One patient had an abdominally placed ICD; in other patients the device was implanted pectorally. The programming was left to the individual investigator's choice; however, due to the long time span of the study, in many of the first implants the commonly used setting for VF detection was 12 of 16 intervals.

\section{ICD follow-up}

The patients were routinely followed at one, three and six month(s) after implantation, then every six months regularly, as in the case of shock therapy. The therapy was classified as appropriate if analysis of the onset, stability, QRS morphology (if available), and termination of the tachycardia with ICD therapy suggested the ventricular origin of arrhythmia. Inappropriate shocks were defined as those delivered in the absence of ventricular arrhythmia. Device effectiveness was assessed by the number of patients who had an appropriate defibrillation after ICD implantation: only the first appropriate shock was considered for analysis. Electric storm was defined as $\geq$ three appropriate ICD shocks in $24 \mathrm{~h}$, and clustered ICD therapies as $\geq$ three either appropriate or inappropriate ICD therapies in $24 \mathrm{~h}$.

\section{Statistical analysis}

Statistical calculations were performed using the Statistica PL software (StatSoft, Inc., Tulsa, OK, USA). Data are presented as either mean \pm standard deviation or median (interquartile range), depending on data type and distribution on the interval scale. We analysed the demographic, clinical, and therapy-related data. The clinical data included previous cardiac arrest, documented TdP, prior syncope and recurrent syncope despite $\beta$-blocker treatment, QTc interval in leads II and V5, LQT locus, Schwartz score, family history of SCD, as well as age at first symptoms and at ICD implantation. The therapy-related data included personal history of LQTS treatment ( $\beta$-blockers, pacemakers), $\beta$-blocking therapy after ICD implantation, and ICD-related data (ICD type, R-wave amplitude on intracardiac electrogram, defibrillation threshold).

Normal distributions were verified with W Shapiro-Wilk test. Intergroup differences were examined using Student $\mathrm{t}$ test, Mann-Whitney $U$ test, and the $\chi^{2}$ test or Fisher exact test when necessary. The Kaplan-Meier method was used for the presentation of appropriate and inappropriate ICD intervention-free periods. Log-rank method was used to investigate significant differences between the groups in the time to the first event (appropriate or inappropriate shock). Parameters expressed as continuous data were categorised, and borderline values for each category were as follows: age at first symptoms and at ICD implantation - 40 years, QTc - $500 \mathrm{~ms}$; number of syncopal episodes - three; Schwartz score -4 ; R-wave amplitude $-5 \mathrm{mV}$, defibrilla- 
tion threshold (DFT) - 10 J. Subsequently, the variables with a p-value $<0.05$ in the log-rank analysis were entered in the multivariable Cox regression analysis for proportional hazards assumptions. Significance was assumed at $p<0.05$.

\section{RESULTS}

\section{Patient characteristics}

Baseline characteristics of the 67 patients are shown in Table 1. The mean age at ICD implantation was 31.4 years (range, $12-77$ years). At the time of implantation, 45 (67\%) patients were $\leq 40$, and five patients were $\geq 60$ years old (Fig. 1 ). The median age at first symptom was 24 years (range, $8-77$ years), and the median Schwartz score was 4 (range, 2-7). A total of $22(33 \%)$ patients were treated with $\beta$-blockers before ICD implantation. In 14 patients, drugs were ineffective in preventing syncope or cardiac arrest. Five patients had pacemakers implanted during childhood, but symptoms recurred despite $\beta$-blocker treatment and pacing in four of them. In seven patients, cardiac arrest was complicated by neurological defects.

Six patients were implanted with pacemakers in the years 1985 to 2000 because of multiple syncopal episodes in the course of LQTS in four patients, syncope with sinus bradycardia in one patient (in whom LQTS diagnosis was overlooked), and a strong family history of SCD in a 10-year-old LQTS boy in the year following the death of his nine-year-old brother. There were AAI (three cases), DDD (two cases), and VVI (one case) pacemakers used. None of the patients was diagnosed with atrioventricular (AV) conduction disturbances. All patients were re-implanted with ICDs when the ICD became available as a measure of primary prevention of SCD in Poland, or when they reached secondary prevention criteria (three patients). At ICD implantation left ventricular ejection fraction was normal.

\section{ICD implantation}

A total of 46 (69\%) patients were cardiac arrest survivors. In 21 patients, an ICD was implanted in primary prevention of SCD; in 18 of those 21 patients, it was implanted because of syncope or recurrent syncope despite $\beta$-blocker/pacemaker therapy. Three other patients had strong positive family histories of sudden death in at least two close family members $<40$ years of age.

A dual-chamber device as a first ICD was implanted in $32(48 \%)$ patients. Median DFT at first ICD implant was $10 \mathrm{~J}$ (range, 3-20 J). There were no significant differences in DFT at subsequent ICD reimplantations, except for one case in which high DFT at reimplantation prompted the insertion of a high-energy device.

\section{Follow-up}

The median follow-up was 47.8 months, with a range from one month to 11 years. In $25 \%$ of patients the follow-up was $\leq 25.6$ months, and in $25 \%$ it was $\geq 68.4$ months. One
Table 1. Baseline characteristics (clinical history) of the study patients $(n=67)$

\begin{tabular}{|lc|}
\hline Clinical history: & $59(88)$ \\
Female sex & $24(8-77)$ \\
Age at first cardiac event [years] & $31(12-77)$ \\
Age at implantation [years] & $482 \pm 35$ \\
QTC II [ms] & $483 \pm 30$ \\
QTC V5 [ms] & $4(2-7)$ \\
Schwartz score & $12(18)$ \\
SCD in family history & $7 / 15 / 4$ \\
LQT1/LQT2/LQT3 locus & \\
Indications for ICD: & $46(69)$ \\
Cardiac arrest survivor & $18(27)$ \\
Syncope or recurrent syncope despite treatment & $3(4.5)$ \\
Malignant family history & $23(34)$ \\
$\beta$-blockers before ICD implantation & $6(9)$ \\
Prior pacemaker implantation & \\
ICD parameters at implantation: & $35 / 32$ \\
ICD type (single-/dual-chamber) & $10(2-30)$ \\
R-wave [ms] & $10(3-20)$ \\
Defibrillation threshold [J] &
\end{tabular}

Data are presented as number (percentage), mean \pm standard deviation, or median (interquartile range) depending on data type and distribution on the interval scale. ICD — implantable cardioverter-defibrillator; LQTS — long QT syndrome; SCD — sudden cardiac death; QTc - corrected QT interval

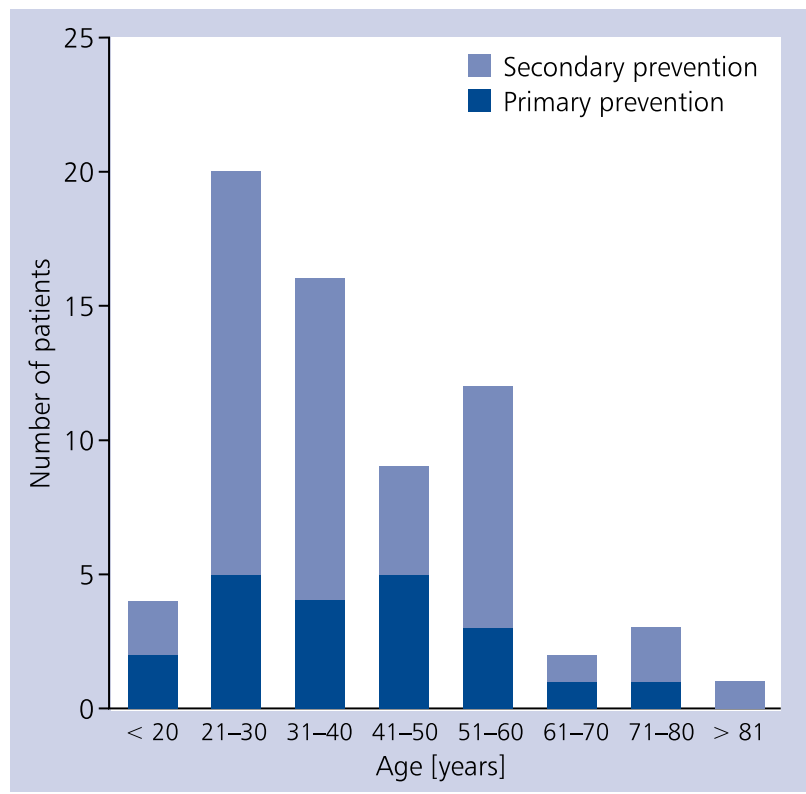

Figure 1. Age at implantable cardioverter-defibrillator implantation in long QT syndrome patients implanted in the primary and secondary prevention of sudden cardiac death 


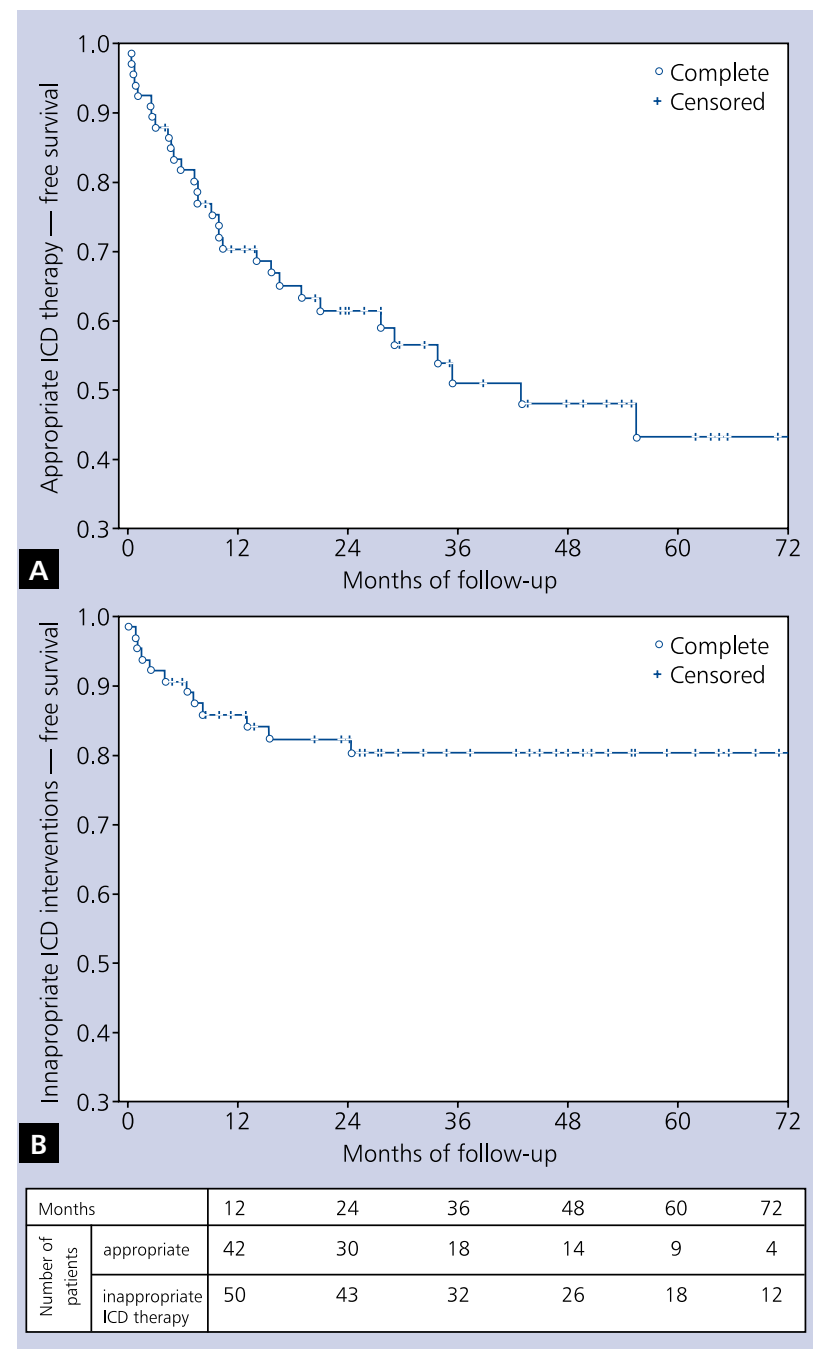

Figure 2. Kaplan-Meier curves for appropriate (A) and inappropriate (B) implantable cardioverter-defibrillator (ICD) therapy-free survival analysis patient was lost to follow-up and one other patient did not attend routine check-ups and, after receiving the necessary explantation of an ICD system because of lead failure and battery depletion, refused ICD reimplantation and further treatment. All other patients presented for scheduled follow-up visits.

\section{Appropriate ICD therapies}

Appropriate ICD therapies occurred in 30 patients (45\%) (Fig. 2). In this group, 23 (77\%) patients were cardiac arrest survivors, whereas the remaining patients were implanted due to syncope (six) or malignant family history (one). The majority of patients $(80 \%)$, including six primary prevention patients, took $\beta$-blockers at the time of the arrhythmic episode triggering ICD intervention. Patients with appropriate therapy had 254 ICD discharges (range, 1-66 therapies); median time to first ICD therapy was nine months (range, 0.4-55 months). Characteristics of patients with and without appropriate ICD therapies are presented in Table 2.

Risk factors for appropriate ICD therapy in survival analysis were: (i) recurrent syncope despite $\beta$-blocker treatment before ICD implantation, (ii) pacemaker therapy before ICD implantation, (iii) single-chamber ICD, and (iv) noncompliance to $\beta$-blocker therapy. However, in the Cox analysis, only the last three were confirmed to be independent risk factors (Table 3).

Table 3. Risk factors for appropriate implantable cardioverter-defibrillator (ICD) therapy

\begin{tabular}{|lccc|}
\hline Variable & Hazard ratio & $95 \% \mathbf{C l}$ & $\mathbf{p}$ \\
\hline Pacemaker before ICD & 6.43 & $2.18-18.91$ & 0.0007 \\
ICD-VVI & 2.51 & $1.12-5.62$ & 0.0254 \\
Concomitant BB therapy & 0.19 & $0.06-0.65$ & 0.0080 \\
\hline
\end{tabular}

Only parameters that were shown to be significant in survival analysis were included in Cox analysis. BB - b-blocker; $\mathrm{Cl}$ - confidence interval

Table 2. Characteristics of patients with and without appropriate implantable cardioverter-defibrillator (ICD) therapies

\begin{tabular}{|lccc} 
& Appropriate ICD therapy & No ICD therapy & p \\
\hline Clinical history: & & & \\
Female sex & $27(90)$ & $32(87)$ & 0.72 \\
Age at first cardiac event [years] & $20(8-77)$ & $27(9-72)$ & 0.58 \\
Age at implantation [years] & $30(16-77)$ & $43(12-72)$ & 0.48 \\
QTC II [ms] & $477 \pm 36$ & $485 \pm 35$ & 0.44 \\
QTC V5 [ms] & $482 \pm 37$ & $484 \pm 24$ & 0.82 \\
Schwartz score & $4(3-7)$ & $4(2-6)$ & 0.75 \\
SCD in family history & $5(17)$ & $6(16)$ & 0.61 \\
LQT1/LQT2/LQT3 locus & $1 / 7 / 0$ & $6 / 8 / 4$ & \\
Indications for ICD: & & & $23(62)$ \\
Cardiac arrest survivor & $23(77)$ & $4(11)$ & 0.20 \\
Syncope or recurrent syncope despite treatment & $11(37)$ & $13(35)$ & 0.013 \\
Malignant family history & $7(23)$ & $23(62)$ & 0.29 \\
Dual-chamber ICD & $9(30)$ & $33(100)$ & 0.0088 \\
$\beta$-blocker treatment & $25(86)$ & 0.043 \\
\hline
\end{tabular}

Data are presented as number (percentage), mean \pm standard deviation or median (interquartile range), depending on data type and distribution. Abbreviations - see Table 1 


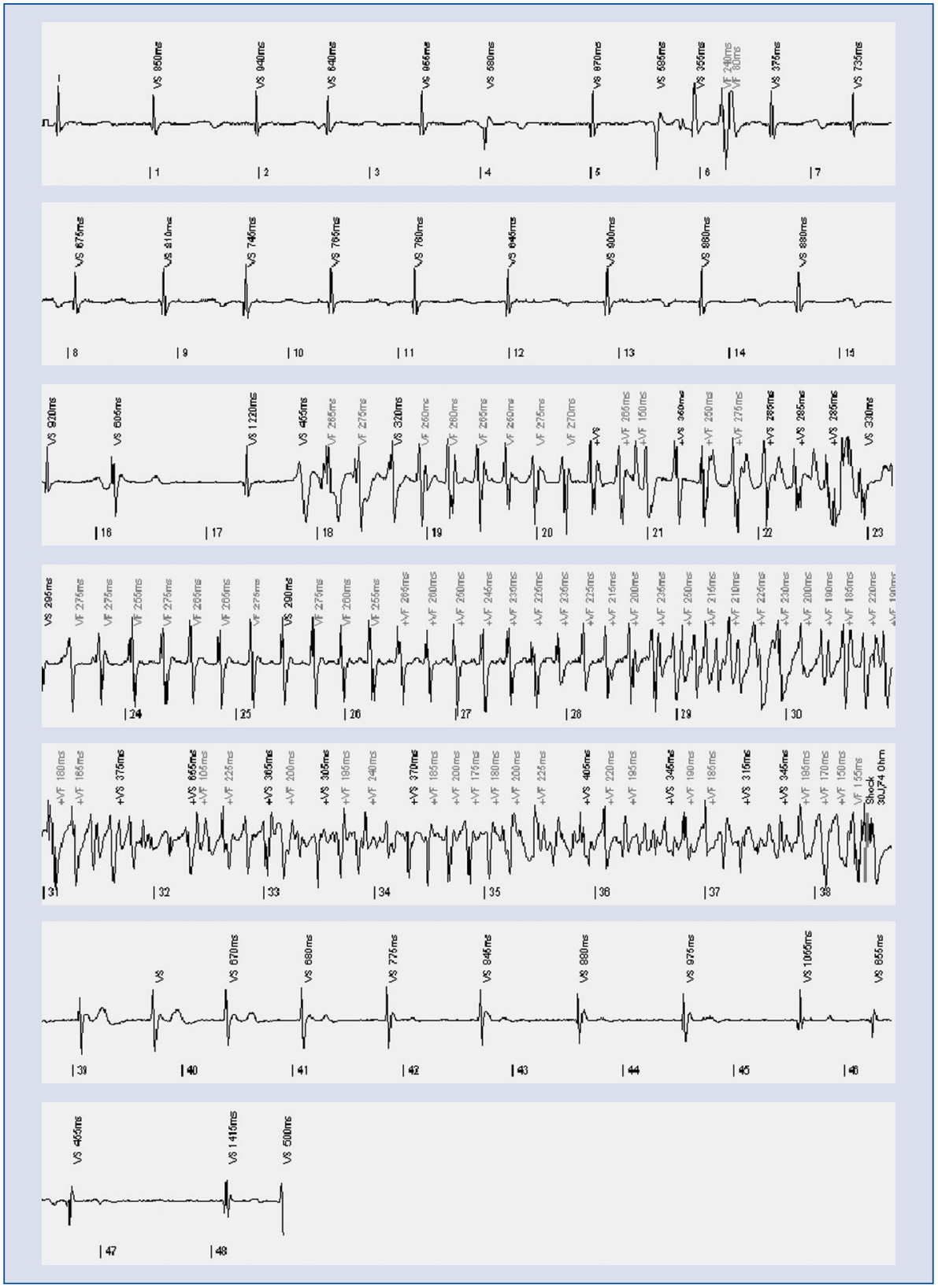

Figure 3. An episode of ventricular tachyarrhythmia in a long QT syndrome patient, terminated by an implantable cardioverter-defibrillator shock

QTc, sex, and locus were not shown to be risk factors. The analysis of independent risk factors for secondary prevention patients showed that the presence of the pacemaker prior to an ICD implantation was the only independent risk factor, with hazard ratio 5.29 and $95 \%$ confidence interval $(\mathrm{Cl})$ $1.032-27.09 ; p=0.046$. However, due to the low number of pacemaker patients the $\mathrm{Cl}$ is very wide. When the ICD patients with an appropriate shock were compared to those without, there were no differences in age, sex, QTc interval, age at first LQTS symptom, Schwartz score, or personal and family history of SCD. However, patients with appropriate ICD therapy had more syncopal episodes before ICD implantation despite $\beta$-blocking treatment ( $37 \%$ vs. $11 \%, \mathrm{p}=0.013$ ), they were less compliant to $\beta$-blockers after ICD implantation ( $86 \%$ vs. $100 \%, p=0.043$ ), and they more frequently had single-chamber ICDs ( $70 \%$ vs. $38 \%, p=0.0088)$ (Table 2$)$.

\section{Inappropriate therapies}

In 14 (21\%) patients, 48 inappropriate therapies occurred (Fig. 3). Of these 14 patients, 10 were cardiac arrest survivors. 
Table 4. Data of patients with inappropriate implantable cardioverter-defibrillator (ICD) therapy

\begin{tabular}{lccccc|} 
Initials & Age at implantation & Genotype & ICD type & $\begin{array}{c}\text { Trigger for inappropriate } \\
\text { therapy }\end{array}$ & $\begin{array}{c}\beta \text {-blocking } \\
\text { therapy }\end{array}$ \\
\hline M.J. & 16 & - & D & SR & + \\
M.P. & 20 & HERG & D & SR & + \\
S.M. & 35 & SCN5A & D & SR & + \\
H.Z. & 71 & HERG & V & AF & + \\
P.R. & 17 & KCNQ1 & V & TWO & + \\
E.S. & 21 & HERG & D & SR, myopotentials & + \\
J.S. & 37 & - & D & SR & + \\
E.G. & 45 & - & D & TWO & - \\
H.K. & 29 & - & D & Ventricular lead failure & + \\
E.P. & 35 & - & D & SR & + \\
A.B. & 18 & - & D & AF & + \\
K.M. & 20 & - & V & TWO & + \\
Z.Z. & 56 & - & V & AF & + \\
H.K. & 48 & KCNQ1 & V then D & AF, nsVT & + \\
\hline
\end{tabular}

$\mathrm{AF}$ - atrial fibrillation; D — dual-chamber ICD; nsVT — non-sustained ventricular tachycardia; SR — sinus rhythm; TWO — T-wave oversensing; $\mathrm{V}$ - single-chamber ICD

Inappropriate interventions were triggered by fast sinus rhythm (SR; six), atrial fibrillation (AF; four), T-wave oversensing (three), myopotentials (one), and lead failure (one patient). In one case, the ICD fired for non-sustained polymorphic VT; the majority of patients $(92 \%)$ were on $\beta$-blockers at the time of inappropriate ICD firing. The median time to first inappropriate therapy was 3.6 months (range, one day - 24.3 months). No independent risk factors for inappropriate therapy were found. Data for patients with inappropriate discharges are shown in Table 4.

\section{Freedom from (in)appropriate shocks and overall survival}

In an attempt to estimate the risk of recurrent life-threatening ventricular arrhythmias and thus the potential impact of ICD on survival, freedom from appropriate ICD therapy was estimated (Fig. 2). No difference in the frequency of first ICD therapy depending on indications for ICD or sex was found. There were no differences in time to first appropriate and inappropriate ICD therapy.

\section{Primary prevention of sudden death}

Among 18 patients in whom an ICD was implanted because of syncope, six had appropriate ICD discharges despite $\beta$-blocker therapy. Although significant family history was the sole indication for ICD insertion in three patients representing two families with particularly malignant phenotype, primary prevention patients more frequently had family history of sudden death $(33.3 \%$ vs. $9.5 \%, p=0.017)$, as compared to patients who received an ICD in secondary prevention of
SCD. Primary prevention patients also had more syncopal spells before ICD implantation (90\% vs. $65 \%, p=0.027$ ).

\section{Clustered ICD therapies}

Six (8.9\%) patients presented with 14 episodes of clustered ICD shocks. Arrhythmic storm ( $\geq$ three episodes of VT/VF within $24 \mathrm{~h}$ ) occurred in five $(7.7 \%)$ patients, recurring from two to three times in four of them during the period under study. Other episodes of clustered ICD shocks were caused by AF (three), T-wave oversensing (one), or lead failure (one). Interestingly, in a female patient in her sixth decade of life, six episodes of multiple ICD shocks occurred. These were triggered initially by ventricular tachyarrhythmias, but, within a few years, paroxysmal AF developed, which is known to cause clustered ICD shocks. After unsuccessful treatment with AV node blocking agents, AV junction ablation was performed, and a dual-chamber ICD was implanted in the patient, revealing several episodes of AF and a separate run of ventricular tachyarrhythmia.

\section{Complications and re-interventions}

Early complications, which occurred during the first month in nine $(13 \%)$ patients, were pneumothorax in four, dislodgment of atrial and/or ventricular lead in six, and infection of the ICD pocket in one patient. Late complications (occurring later than one month after implantation) included ICD malfunction (14 cases of early battery depletion, system errors in 13 patients), lead failure requiring reimplantation of a new lead (13 cases in nine patients), pocket and/or lead infection that required both lead and generator replacement (three 
patients), and high defibrillation threshold at reimplantation. Failure of the defibrillation lead was recognised in six patients, and dysfunction of the atrial lead or both leads was recognised in one and two patients, respectively. In one subject, the defibrillation lead was broken twice. There was one case of late dislodgement of the defibrillation lead with a perforation of the right ventricle, which was diagnosed three years after ICD implantation, being the cause of thoracic surgery in a 19-year-old man. Overall, 38 (57\%) patients had at least one complication, such as inappropriate ICD intervention, clustered ICD therapy, dislodgement or malfunction of an ICD lead, or an infection-related event (scheduled ICD replacements were not included). A total of 108 ICDs were implanted in the 67 patients studied: one device in 34 patients, two in 26 patients, three in five patients, and four in three patients. In 19 (28\%) patients, 21 procedures were performed due to elective replacement indications. In four patients, ICD was upgraded to ICD-DDD, whereas two patients, who had dual-chamber ICDs previously, received the ICD-VVI due to atrial lead failure or infective endocarditis.

\section{DISCUSSION}

The present study is the first multicentre study to analyse the outcome, complications, and risk factors of ICD therapy in LQTS patients in Poland. It demonstrated that ICD is effective in nearly half of patients; however, the early and late complication rates during a median follow-up of 48 months are high. The observed frequency of re-interventions and inappropriate discharges provide evidence against a more aggressive implanting approach.

\section{Indications for ICD implantations}

Contrary to other registries, in which cardiac arrest survivors constituted less than $50 \%$ of implanted LQTS patients, $69 \%$ of our patients had cardiac arrest before ICD implantation [4-7]. This is probably the reason for the higher rate of appropriate ICD shocks, which occurred in $45 \%$ of patients in our study. It is likely, however, that many of these shocks could be prevented by longer detection settings.

Among primary prevention patients, three were implanted with ICDs because of a strong family history (e.g. death of five family members $<40$ years of age within one family). None of these patients experienced appropriate ICD shocks during long-term follow-up, while one of them suffered from inappropriate shocks caused by an ICD lead failure. In the United States a strong family history was considered an indication for an ICD implantation. However, in LQTS, the clinical usefulness of family history has never been confirmed (as opposed to hypertrophic cardiomyopathy) [8-10]. Our observation confirms that, even in patients with very severe family history, empathy-driven decisions on ICD implantations should be avoided.

\section{Appropriate discharges}

In our study, ICD therapy was shown to be effective, delivering one or more potentially lifesaving therapies in $45 \%$ of patients. Interestingly, when compared to other genetically determined arrhythmogenic disorders, the observed rate of ICD therapies is the highest [2-5], except for patients with arrhythmogenic right ventricular cardiomyopathy, in whom similar ICD intervention rates have been shown [11]. The frequent appropriate ICD therapies observed in our study may be related to the relatively high number of human Ether-a-go-go-related Gene (hERG) patients who used to have numerous cardiac events despite $\beta$-blocker therapy [12]. The predominance of secondary prevention patients, who usually were probands, may also be responsible for the rate of ICD interventions, because probands were shown to be more symptomatic [13]. Noncompliance with $\beta$-blockers may be another reason for high arrhythmia rate because only $87 \%$ of patients took $\beta$-blockers at the time of the arrhythmic episode, which demonstrates the importance of educating LQTS patients on how to be compliant with $\beta$-blockers, even if an ICD is implanted. In contrast, it is well appreciated that $\mathrm{TdP}$ is not invariably fatal, and, even if the longest possible detection time is programmed, it cannot be excluded that arrhythmia would terminate spontaneously if not treated.

We tried to identify the potential predictors of appropriate shocks. However, the known risk factors of SCD, including sex and QTC [14], did not predict the occurrence of appropriate shocks, whereas the number of genotyped patients was too small to impact statistics. In the Cox analysis pacemaker therapy before ICD implantation, single-chamber ICD, and noncompliance to $\beta$-blocker therapy after implantation were shown to be independently associated with the risk of appropriate ICD therapy. Before the ICD era, pacemakers were used mainly in symptomatic LQTS patients or were implanted instead of ICDs in highly symptomatic children. This finding may show that the relatively more symptomatic course of the LQTS during childhood still confers a risk in adults. When the relation between single-chamber ICD and appropriate ICD shocks is considered, a pro-arrhythmic effect of $\mathrm{V} V \mathrm{I}$ pacing in LQTS patients cannot be excluded. Because an analysis of the initiating mechanism was not available in our study, the clinical significance of this assumption requires further investigation. However, since episodes of $\mathrm{TdP}$ are frequently triggered by short-long-short sequences, this finding may point to a lack of rhythm stabilisation in VVI patients.

Family history of SCD, while important for establishing the diagnosis, is not considered helpful for risk stratification [10]. As we have shown, malignant family history strongly impacts decision-making about ICD insertion; as many as $33.3 \%$ of primary prevention patients had a family history of sudden death, compared to $9 \%$ of secondary prevention patients $(p=0.02)$. Furthermore, in three patients, significant family history was the only indication for ICD implantation, whereas, in one of these patients, multiple appropriate ICD shocks occurred during follow-up.

It is important to stress that time to a first appropriate discharge was up to 55 months. Considering the average life 
of ICD batteries, this finding is of clinical relevance for patients who may suffer from ICD-related adverse effects and reject ICD replacement on the basis of its alleged futility.

\section{Avoidance of ICD shocks}

The percentage of patients receiving inappropriate therapy in this study was $21 \%$, which is similar to the data observed in other young ICD populations [11, 15, 16]. Inappropriate shocks were triggered mainly by fast SR, atrial arrhythmias, and T-wave oversensing. Johnson et al. [17] documented that AF occurs in around $2 \%$ of LQTS patients, and in our study, AF triggered the ICD response in $6 \%$ of patients. The high rate of inappropriate therapies due to the oversensing of SR or AF demonstrates further that ICD therapy should be combined with $\beta$-blocker treatment.

In the context of LQTS, the programming of therapeutic zones and therapies constitutes an important concern. The PainFREE trial has shown that activating antitachycardia pacing (ATP) for fast VT is effective in $81 \%$ of the episodes, thereby significantly reducing the number of appropriate shocks [18]. It is unknown, however, whether ATP can convert polymorphic VT such as TdP, although it seems unlikely because of the rapid rate and suspected disorganisation of TdP. The question of how many shocks could be prevented by using ATP in a VF window merits further investigation. Moreover, some pacing algorithms used in modern pacemakers may have proarrhythmic effects [19-21]. Thus, universal recommendations on ICD and pacemaker programming are needed.

\section{Electrical storms}

It is well appreciated that, in LQTS, an adrenergic drive during exercise or strong emotions may trigger life-threatening arrhythmias [22]. It was speculated that ICD shock via catecholamine release may increase susceptibility to arrhythmias and subsequent ICD shocks leading to electrical storms. Indeed, this phenomenon is observed in LQTS patients. In the study by Mönning et al. [5], as many as $18.5 \%$ of 27 ICD patients experienced electrical arrhythmic storms despite $\beta$-blocker therapy. In our study, arrhythmic storms were less prevalent, occurring in $9 \%$ of patients. Notably, electrical storms tended to recur in the same patients, showing that a specific genetic susceptibility may underlie these adverse effects.

\section{Complications}

Our study confirms that lead longevity is one of the major problems in patients with a long life expectancy, which is in accordance with the results of other studies of young patients [15, 16, 23, 24]. In young and active patients, long life expectancy results in increased numbers of lead extractions and replacements, reaching $13.4 \%$ of patients in our study. This is most clearly shown in our patient in whom lead failure required as many as three defibrillation leads to be implanted. Not only does lead malfunction increase the number of surgical re-interventions, but it may also result in life-threatening conditions such as ICD system infection or right ventricular perforation, increasing significantly the morbidity of LQTS-ICD patients (in our study, $4.5 \%$ and $1.5 \%$, respectively).

Another important concern is the high frequency of ICD reimplantations, which were caused either by expected end of life, ICD malfunction, ICD system and/or pocket infection, or high DFT, and which occurred in 34 (51\%) patients. In the future, improvement of the battery's technical capability and prevention of inappropriate shocks may reduce the need for re-interventions, which may subsequently decrease the accompanying complications. Of particular importance is a cluster of device recalls that occurred during the period under study, increasing the rate of device re-interventions. This is not expected to be repeated in future years; however, it underscores the concern that this therapy is not without unexpected risk. Although the number of unnecessary ICD shocks and reimplantation procedures may be lowered by modern programming and increased longevity of newer ICDs, other adverse events are less likely to be reduced. In some, but not all, LQTS patients a subcutaneous ICD may be an option to minimise the risk of complications [25, 26].

This study is based on an observational multicentre registry and is subject to all the inherent limitations. Although every effort was made to collect the data in a uniform and thorough manner, some measurement bias may have occurred. Furthermore, device programming was not identical in all centres. Additionally, the relatively small number of patients limited our ability to identify predictors of appropriate and inappropriate ICD therapies. Another important concern is the deleterious psychological impact of ICD implantation on young patients. Anxiety and psychological distress are recognised to be more frequent in young ICD recipients; however, they were not assessed systematically in the study. Lastly, because genetic screening of LQTS genes is not reimbursed in Poland, data on LQTS genotype were available only in a minority of patients and were not included in the study.

In conclusion, although ICD proved to be effective in $45 \%$ of LQTS patients, this therapy was associated with high complication rates. In our opinion, the present study does not support a more liberal use of ICD in LQTS patients. By providing explicit data regarding the risks and benefits of long-term ICD therapy, our findings may be useful in informing patients and physicians who need to make decisions regarding ICD implantation, particularly in the primary prevention of SCD.

Funding: This work was supported by the Polish Scientific Committee grant no. 2P05B09826 (to A.L., A.Z.K.).

\section{Conflict of interest: none declared}

\section{References}

1. Priori S, Blomström-Lundqvist C, Mazzanti A, et al. 2015 ESC Guidelines for the management of patients with ventricular arrhythmias and the prevention of sudden cardiac death. Eur Heart J. 2015; 36(41): 2793-2867, doi: 10.1093/eurheartj/ehv316. 
2. Kaufman ES. Arrhythmic risk in congenital long QT syndrome. J Electrocardiol. 2011; 44(6): 645-649, doi: 10.1016/j.jelectrocard.2011.07.023, indexed in Pubmed: 21907997.

3. Schwartz PJ, Moss AJ, Vincent GM, et al. Diagnostic criteria for the long QT syndrome. An update. Circulation. 1993; 88(2): 782-784, indexed in Pubmed: 8339437.

4. Zareba W, Moss A, Daubert J, et al. Implantable cardioverter defibrillator in high-risk long QT syndrome patients. J Cardiovasc Electrophysiol. 2003; 14(4): 337-341, doi: 10.1046/j.1540-8167 .2003.02545.x.

5. Mönnig G, Köbe J, Löher A, et al. Implantable cardioverter-defibrillator therapy in patients with congenital long-QT syndrome: a long-term follow-up. Heart Rhythm. 2005; 2(5): 497-504, doi: 10.1016/j.hrthm.2005.02.008, indexed in Pubmed: 15840474 .

6. Horner JM, Kinoshita M, Webster TL, et al. Implantable cardioverter defibrillator therapy for congenital long QT syndrome: a single-center experience. Heart Rhythm. 2010; 7(11): 1616-1622, doi: 10.1016/j.hrthm.2010.08.023, indexed in Pubmed: 20816872.

7. Schwartz PJ, Spazzolini C, Priori SG, et al. Who are the long-QT syndrome patients who receive an implantable cardioverter-defibrillator and what happens to them?: data from the European Long-QT Syndrome Implantable Cardioverter-Defibrillator (LQTS ICD) Registry. Circulation. 2010; 122(13): 1272-1282, doi: 10.1161/CIRCULATIONAHA.110.950147, indexed in Pubmed: 20837891.

8. Kaufman ES. Saving lives in congenital long QT syndrome: who benefits from implantable cardioverter defibrillator therapy? J Cardiovasc Electrophysiol. 2003; 14(4): 342-343, indexed in Pubmed: 12741702.

9. Epstein AE, Dimarco JP, Ellenbogen KA, et al. ACC/AHA/HRS 2008 Guidelines for Device-Based Therapy of Cardiac Rhythm Abnormalities: a report of the American College of Cardiology/American Heart Association Task Force on Practice Guidelines (Writing Committee to Revise the ACC/AHA/NASPE 2002 Guideline Update for Implantation of Cardiac Pacemakers and Antiarrhythmia Devices): developed in collaboration with the American Association for Thoracic Surgery and Society of Thoracic Surgeons. Circulation. 2008; 117(21): e350-e408, doi: 10.1161/CIRCUALTIONAHA.108.189742, indexed in Pubmed: 18483207.

10. Kaufman ES, McNitt S, Moss AJ, et al. Risk of death in the long QT syndrome when a sibling has died. Heart Rhythm. 2008; 5(6): 831-836, doi: 10.1016/j.hrthm.2008.02.029, indexed in Pubmed: 18534367.

11. Wichter T, Paul M, Wollmann C, et al. Implantable cardioverter/defibrillator therapy in arrhythmogenic right ventricular cardiomyopathy: single-center experience of long-term follow-up and complications in 60 patients. Circulation. 2004; 109(12): 1503-1508, doi: 10.1161/01.CIR.0000121738.88273.43, indexed in Pubmed: 15007002.

12. Priori SG, Napolitano C, Schwartz PJ, et al. Association of long QT syndrome loci and cardiac events among patients treated with beta-blockers. JAMA. 2004; 292(11): 1341-1344, doi: 10.1001/jama.292.11.1341, indexed in Pubmed: 15367556.

13. Kimbrough J, Moss AJ, Zareba W, et al. Clinical implications for affected parents and siblings of probands with long-QT syndrome. Circulation. 2001; 104(5): 557-562, indexed in Pubmed: 11479253.

14. Napolitano C, Priori SG, Schwartz PJ, et al. Risk stratification in the long-QT syndrome. N Engl J Med. 2003; 348(19): 1866-
-1874, doi: 10.1056/NEJMoa022147, indexed in Pubmed: 12736279.

15. Olde Nordkamp LRA, Wilde AAM, Tijssen JGP, et al. The ICD for primary prevention in patients with inherited cardiac diseases: indications, use, and outcome: a comparison with secondary prevention. Circ Arrhythm Electrophysiol. 2013; 6(1): 91-100, doi: 10.1161/CIRCEP.112.975268, indexed in Pubmed: 23275262.

16. Sacher F, Probst V, Maury P, et al. Outcome after implantation of a cardioverter-defibrillator in patients with Brugada syndrome: a multicenter study-part 2. Circulation. 2013; 128(16): 1739-1747, doi: 10.1161/CIRCULATIONAHA.113.001941, indexed in Pubmed: 23995538.

17. Johnson JN, Tester DJ, Perry J, et al. Prevalence of early-onset atrial fibrillation in congenital long QT syndrome. Heart Rhythm. 2008; 5(5): 704-709, doi: 10.1016/j.hrthm.2008.02.007, indexed in Pubmed: 18452873.

18. Wathen MS, DeGroot PJ, Sweeney MO, et al. Prospective randomized multicenter trial of empirical antitachycardia pacing versus shocks for spontaneous rapid ventricular tachycardia in patients with implantable cardioverter-defibrillators: Pacing Fast Ventricular Tachycardia Reduces Shock Therapies (PainFREE Rx II) trial results. Circulation. 2004; 110(17): 2591-2596, doi: 10.1161/01. CIR.0000145610.64014.E4, indexed in Pubmed: 15492306.

19. Rudic B, Tülümen E, Borggrefe M, et al. Proarrhythmic effect of "reverse mode switch" in a patient with long-QT syndrome. J Cardiovasc Electrophysiol. 2014; 25(10): 1133-1134, doi: $10.1111 /$ jce.12459.

20. Di Cori A, Zucchelli G, Romano S, et al. Proarrhythmic effect of pacing mode reprogramation in a patient with a congenital long-QT syndrome. J Cardiovasc Electrophysiol. 2013; 24(11): 1304-1305, doi: 10.1111/jce.12179, indexed in Pubmed: 23758507.

21. Schellevis MM, VAN Halm VP. Ventricular fibrillation due to automated atrial threshold testing in a patient with an implantable cardioverter defibrillator. Pacing Clin Electrophysiol. 2015; 38(11): 1355-1358, doi: 10.1111/pace.12639, indexed in Pubmed: 25851701

22. Schwartz P, Priori S, Spazzolini C, et al. Genotype-Phenotype correlation in the long-QT syndrome. Circulation. 2001; 103(1): 89-95, doi: 10.1161/01.cir.103.1.89.

23. Olde Nordkamp LRA, Postema PG, Knops RE, et al. Implantable cardioverter-defibrillator harm in young patients with inherited arrhythmia syndromes: A systematic review and meta-analysis of inappropriate shocks and complications. Heart Rhythm. 2016; 13(2): 443-454, doi: 10.1016/j.hrthm.2015.09.010, indexed in Pubmed: 26385533

24. Gaba P, Bos J, Cannon B, et al. Implantable cardioverter-defibrillator explantation for overdiagnosed or overtreated congenital long QT syndrome. Heart Rhythm. 2016; 13(4): 879-885, doi: 10.1016/j.hrthm.2015.12.008.

25. Botto GL, Forleo GB, Capucci A, et al. 'AIAC S-ICD Why Not' Survey Investigators. The Italian subcutaneous implantable cardioverter-defibrillator survey: S-ICD, why not? Europace. 2017; 19(11): 1826-1832, doi: 10.1093/europace/euw337, indexed in Pubmed: 28011803.

26. Ptaszyński P, Grabowski M, Kowalski O, et al. [Subcutaneous implantable cardioverter-defibrillator in prevention of sudden cardiac death in Poland - opinion paper endorsed by the Polish Cardiac Society Working Group on Heart Rhythm]. Kardiol Pol. 2017; 75(10): 1057-1060, doi: 10.5603/KP.2017.0196, indexed in Pubmed: 29057442.

Cite this article as: Zienciuk-Krajka A, Sterliński M, Filipecki A, et al. Implantable cardioverter-defibrillators in patients with long QT syndrome: a multicentre study. Kardiol Pol. 2018; 76(12): 1687-1696, doi: 10.5603/KP.a2018.0177. 


\section{WHAT IS NEW?}

No randomised clinical trial has ever been conducted on patients with implantable cardioverter-defibrillators (ICDs) and long QT syndrome (LQTS), thus registry data are still of importance to help in risk stratification and to improve treatment options. In our multicentre study of primary and secondary prevention in LQTS patients, risk factors for appropriate ICD therapy were: recurrent syncope despite $\beta$-blockers before ICD implantation, pacemaker therapy before ICD implantation, a single-chamber ICD, and noncompliance to $\beta$-blockers. Inappropriate therapies were triggered by fast sinus rhythm, atrial fibrillation, and T-wave oversensing, however, no predictors of inappropriate shocks were identified. Importantly, in the majority of patients at least one adverse event occurred. By providing data regarding the risk and benefit of ICD therapy, our findings may be useful in informing patients and physicians who need to make decisions regarding ICD implantation. 